\title{
Growth of Anisotropic Platinum Nanostructures Catalyzed by Gold Seed Nanoparticles
}

\author{
Zheng Fang, Yuliang Zhang, Feifei Du, and Xinhua Zhong ( $₫)$ \\ Laboratory for Advanced Materials, Department of Chemistry, East China University of Science \& Technology, Shanghai 200237, China \\ Received: 23 April 2008/ Revised: 7 June 2008/Accepted: 5 August 2008 \\ CTsinghua Press and Springer-Verlag 2008. This article is published with open access at Springerlink.com
}

\begin{abstract}
This paper reports an effective method for the synthesis of platinum nanostructures with anisotropic morphologies by decomposition of platinum dichloride in oleylamine at intermediate temperatures catalyzed by gold seed nanoparticles. A small quantity of spherical gold nanoparticles formed in situ was used to trigger the nucleation and anisotropic growth of the Pt nanocrystals. By varying the amount of gold seed nanoparticles, porous flower-like, irregular polyhedron-shaped, multi-branched rod shaped, and caterpillarlike Pt nanostructures were produced in high yields at $190-240{ }^{\circ} \mathrm{C}$ in reaction times of a few minutes. Control of morphology under different conditions has been systematically studied and a kinetically controlled induced growth mechanism has been proposed.
\end{abstract}

\section{KEYWORDS}

Platinum nanostructure, gold seed nanoparticles, synthesis

\section{Introduction}

Nanocrystals have attracted great interest due to their fundamental size- and shape-dependent properties and their many important potential technological applications [1-6]. In the past decade, intensive effort has been devoted to the design and synthesis of uniformly sized nanocrystals with well-defined anisotropic morphologies, because the morphology (including dimensionality and shape) of most nanostructures can effectively tune their intrinsic chemical and physical properties [7-9]. Nanostructured platinum is an example of considerable interest for many industrial applications due to its extraordinary properties [10]. For example, it serves as a catalyst in the production of hydrogen from methane, in the reduction of pollutant gases in automobile exhaust, and particularly in the direct methanol fuel cell (DMFC) [11-14]. It has been established that the catalytic reactivity of platinum nanostructures depends highly on the morphology of the nanoparticles $[15,16]$, and therefore the design and synthesis of platinum nanoparticles with wellcontrolled shapes and sizes are of critical importance, especially in the field of catalysis.

For these reasons, in the past decade much effort has been devoted to the fabrication of platinum nanostructures with monodisperse sizes and welldefined morphologies. Platinum nanostructures are generally prepared through the reduction of $\mathrm{Pt}(\mathrm{IV})$ or $\mathrm{Pt}(\mathrm{II})$ precursors in solution phase by reducing agents such as alcohols [17-19], sodium borohydride

Address correspondence to zhongxh@ecust.edu.cn 
[20-23], or hydrogen [24, 25] in the presence of organic molecules or polymers as capping agents or morphology-directing reagents. The resultant $\mathrm{Pt}$ nanoparticles synthesized via this route are usually spherical or have undefined facets. An alternative approach is to use soft templates [26-28] (such as micelles) or hard templates [29-34] (such as mesoporous silica) to direct the formation of platinum nanocrystals with anisotropic morphologies. By this route, the resulting morphology is mainly limited to one-dimensional nanorods or nanotubes. Recently, Xia and coworkers exploited a novel route to tune the morphology of platinum nanocrystals, where inorganic species (such as $\mathrm{NaNO}_{3}$ or the $\mathrm{Fe}(\mathrm{II}) / \mathrm{Fe}$ (III) redox pair) were used to manipulate the reduction kinetics of platinum precursors in a solution phase chemical reduction process for the production of platinum nanoparticles and a series of new morphologies were obtained [35-39].

Another common solution phase-based approach to the anisotropic growth of nanostructures is the solution-liquid-solid (SLS) method [40], the solution counterpart of the vapor-liquid-solid (VLS) growth method. Both methods ultimately rely on metal catalyst particles to promote one-dimensional (mainly nanowire) nanostructure growth. However, in the case of the SLS method, the metal nanocrystals must have a melting point capable of being attained by solution chemistry. Catalysts are therefore restricted to common low-melting metals such as In, Sn, Bi, and Ga [40-46]. In contrast to the SLS method, where metal catalyst particles are in the molten liquid state, some noble metal particles with high boiling point (such as $\mathrm{Au}, \mathrm{Ag}$, or Pd) can also be used to trigger the heterogeneous nucleation and growth of a variety of anisotropic semiconductor and metallic nanostructures [47-54]. In these cases, the seed metal particles are not molten under the reaction conditions.

Herein we report the preparation of a variety of anisotropic platinum nanostructures with high yields through an induced anisotropic growth process at temperatures below that required for the homogeneous nucleation and growth of $\mathrm{Pt}$ nanoparticles from platinum dichloride. A small quantity of spherical gold nanoparticles formed in situ by decomposition of gold trichloride $\left(\mathrm{AuCl}_{3}\right)$ at a relatively low temperature is used to trigger formation of anisotropic platinum nanostructures at a higher temperature. The resulting platinum nanostructures have special structural characteristics which are highly desirable and potentially technologically important. Furthermore, access to materials with such well-defined complex morphologies should pave the way for exploring the mechanism of morphology control in the crystal formation process.

\section{Experimental}

\subsection{Materials}

Gold trichloride $\left(\mathrm{AuCl}_{3}, 99.9 \%\right)$, platinum dichloride $\left(\mathrm{PtCl}_{2}, 99 \%\right)$, oleic acid (OA, 95\%), oleylamine (OAm, 90\%) and 1-octadecene (ODE, 90\%) were purchased from Aldrich. All chemicals were used as received. All solvents (hexane, toluene, acetonitrile) were used without further purification.

\subsection{Synthesis of Pt nanocrystals}

In a typical reaction, $25 \mu \mathrm{L}$ of $0.03 \mathrm{~mol} / \mathrm{L} \mathrm{AuCl}_{3}$ stock solution in acetonitrile was loaded in a $50 \mathrm{~mL}$ threenecked round-bottom flask and the acetonitrile was pumped off. $\mathrm{PtCl}_{2}(13.3 \mathrm{mg}, 0.05 \mathrm{mmol})$ and OAm $(5.0 \mathrm{~mL})$ were then added to the flask, and the mixture was degassed at $50{ }^{\circ} \mathrm{C}$ under a vacuum of $\sim 1$ mbar for 5 min with magnetic stirring. After one hour, the reaction vessel was filled with argon, and its temperature was raised to $125^{\circ} \mathrm{C}$ and maintained at this temperature for $10 \mathrm{~min}$. At this stage, the original light yellow solution turned wine red, indicating the formation of gold nanoparticles. The temperature was further increased to $190{ }^{\circ} \mathrm{C}$ with a heating rate of $\sim 10{ }^{\circ} \mathrm{C} / \mathrm{min}$ and the mixture was kept at this temperature for 3 min under argon flow. When the temperature reached $\sim 180{ }^{\circ} \mathrm{C}$ the wine red solution turned black (the transformation from wine red to black took less than $10 \mathrm{~s}$ ), indicating the decomposition of the Pt precursor and the formation of Pt nanoparticles. Subsequently, the reaction mixture was cooled to $\sim 60^{\circ} \mathrm{C}$, and toluene $(10 \mathrm{~mL})$ was added. The black precipitate was isolated and purified by centrifugation and washed several times with chloroform and acetone. The resulting 
organic ligand-coated Pt particles are re-dispersible in nonpolar solvents such as chloroform, toluene, or hexane and were used for subsequent measurements without any size selection. The amount of $\mathrm{AuCl}_{3}$ stock solution was varied from $0-500 \mu \mathrm{L}$, while the amounts of all the other reagents were fixed. The final reaction temperature was $190{ }^{\circ} \mathrm{C}$ unless indicated otherwise.

\subsection{Characterization}

The morphologies and dimensions of the products were observed by transmission electron microscopy (TEM) using a Jeol JEM-1400 instrument at an acceleration voltage at $100 \mathrm{kV}$ and high-resolution TEM (HRTEM) images were obtained on a Tecnai F20 instrument operated at $200 \mathrm{kV}$. TEM samples were prepared by depositing a drop of a dilute toluene dispersion of nanocrystals on a carbon filmcoated copper grid. The chemical composition of the Pt nanoparticles was determined using an energydispersive X-ray (EDX) analyser attached to the Tecnai microscope. The purity and structure of the products were confirmed by powder X-ray diffraction (XRD) obtained on a Philips (PW 1820) X-ray diffractometer equipped with graphite monochromatized high intensity $\mathrm{Cu} \mathrm{K} \alpha$ radiation ( $\lambda=1.54178 \AA$ ) $)$. XRD samples were prepared by depositing powdered nanocrystals on a $\mathrm{Si}(100)$ wafer.

\section{Results and discussion}

\subsection{Formation of Au nanodots at $120^{\circ} \mathrm{C}$}

In the synthesis, $\mathrm{AuCl}_{3}$ was added to the OAm together with $\mathrm{PtCl}_{2}$ and the resulting reaction mixture was first held at $120{ }^{\circ} \mathrm{C}$ for $10 \mathrm{~min}$. During this stage, the color of the solution changed to wine red, corresponding to the characteristic color of gold nanoparticles. The platinum precursor does not decompose at $120{ }^{\circ} \mathrm{C}$-according to the literature, platinum precursors only start to decompose at temperatures of around $240{ }^{\circ} \mathrm{C}$ in OAm [55]. In a control experiment, after the $\mathrm{AuCl}_{3}$ and $\mathrm{PtCl}_{2}$ mixture in OAm was kept at $120^{\circ} \mathrm{C}$ for $10 \mathrm{~min}$, the reaction mixture was allowed to cool to $\sim 60{ }^{\circ} \mathrm{C}$ and $5 \mathrm{~mL}$ of both ODE and hexane were added. Repeated extraction purification procedures were carried out on the nanocrystal dispersion in order to isolate the nanocrystals from the unreacted precursors [56]. Methanol was used as the extraction solvent. The nanocrystals remained in the hexane/ODE layer, and the unreacted precursors and excess amine were extracted into the methanol layer. TEM analysis shows that spherical Au NPs with an average size of $5 \mathrm{~nm} \pm 0.5 \mathrm{~nm}$ were formed under the above conditions (TEM images are available in the Electronic Supplementary Material (ESM)). Based on these findings, we can conclude that it is gold nanoparticles that induce the subsequent morphology-controlled growth of platinum nanocrystals. When we used pre-prepared gold nanoparticles instead of the $\mathrm{AuCl}_{3}$ stock solution, similar results were obtained, supporting this hypothesis. For convenience of operation and more accurate control over the amount of $\mathrm{AuCl}_{3}$ used an $\mathrm{AuCl}_{3}$ stock solution in acetonitrile, rather than solid $\mathrm{AuCl}_{3}$ or pre-prepared Au nanoparticles, was used as the precursor for the Au seeds.

\subsection{Synthesis of $\mathrm{Pt}$ nanostructures}

It was found that the temperature of decomposition of the platinum precursor and the formation of platinum nanocrystals (indicated by the appearance of a black color in the reaction solution) varied with the amount (or concentration) of $\mathrm{AuCl}_{3}$ used. When 0-10 $\mu \mathrm{L} \mathrm{AuCl}_{3}$ stock solution was used, the decomposition temperature was around $240^{\circ} \mathrm{C}$, while the temperature decreased to around $180{ }^{\circ} \mathrm{C}$ when the amount of $\mathrm{AuCl}_{3}$ solution was greater than $25 \mu \mathrm{L}$. There was no significant change in the decomposition temperature with a further increase in the amount of $\mathrm{AuCl}_{3}$. Furthermore, the morphologies of the platinum nanostructures obtained depend markedly on the amount of $\mathrm{AuCl}_{3}$ used as discussed in the following section.

\subsection{Influence of the amount of $\mathrm{AuCl}_{3}$ (or the concentration of Au particles) on the morphology of the Pt nanocrystals}

It is reasonable to deduce that different amounts of added $\mathrm{AuCl}_{3}$ correspond to different concentrations of gold nanoparticles, even though there is perhaps not a strictly linear relationship due to the variable

\section{Springer}


particle sizes obtained with different amounts of $\mathrm{AuCl}_{3}$ used. The dimensions and morphology of the Pt nanocrystals changed significantly when the concentration of gold seed nanoparticles was changed. When a small amount of $\mathrm{AuCl}_{3}(10 \mu \mathrm{L}$ of $\mathrm{AuCl}_{3}$ stock solution, containing $3 \times 10^{-4} \mathrm{mmol}$ $\mathrm{AuCl}_{3}$ ) was used in the synthesis, the platinum precursor $\left(\mathrm{PtCl}_{2}\right)$ decomposed at around $240{ }^{\circ} \mathrm{C}$ and porous flower-like platinum nanostructures (Fig. 1(a)) were formed. The porous structure is built up of tens of elongated primary nanoparticles with average dimensions of $\sim 6 \mathrm{~nm} \times 9 \mathrm{~nm}$. A detailed structural characterization was carried out previously on similar particles, where $\mathrm{Pt}\left(\mathrm{NH}_{3}\right)_{2} \mathrm{Cl}_{2}$ was used as $\mathrm{Pt}$ precursor [55]. The decomposition temperature of the platinum precursor and the morphology of the $\mathrm{Pt}$ nanostructures obtained with the addition of small amounts of $\mathrm{AuCl}_{3}$ are very similar to those observed when no $\mathrm{AuCl}_{3}$ is present. This indicates that small amounts of $\mathrm{AuCl}_{3}$ have no profound effect on the nucleation and growth of platinum nanostructures.

When $25 \mu \mathrm{L}$ of the $\mathrm{AuCl}_{3}$ stock solution (containing $7.5 \times 10^{-4} \mathrm{mmol} \mathrm{AuCl}_{3}$ ) was introduced into the reaction system, large faceted irregular polyhedronshaped nanocrystals were formed (Fig. 1(b)). Although the polyhedra have no uniform shape, they have nearly uniform size in the range 5-8 $\mathrm{nm}$. With extension of the reaction time to $30 \mathrm{~min}$, the overall morphology of the polyhedra was retained with a small increase in the particle dimensions, which can be attributed to Ostwald ripening. When the amount of $\mathrm{AuCl}_{3}$ stock solution was increased to $100 \mu \mathrm{L}$ (containing $3 \times 10^{-3} \mathrm{mmol} \mathrm{AuCl}_{3}$ ), isolated multibranched $\mathrm{Pt}$ nanorods with different number of pods were formed (Fig. 1(c)). The branched nanorods exhibit a roughly spherical morphology with diameters averaging about 15 $\mathrm{nm}$. The diameters of each pod on all the branched rods are relatively constant $(4 \mathrm{~nm} \pm 0.5 \mathrm{~nm})$. When nanostructures the amount of $\mathrm{AuCl}_{3}$ was further increased to 250 $\mu \mathrm{L}$ (containing $7.5 \times 10^{-3} \mathrm{mmol} \mathrm{AuCl}_{3}$ ), almost all the nanostructures display a caterpillar shape with a spherical center surrounded by a small number of pods (Fig. 1(d)). The diameter of the spherical center is around $9-11 \mathrm{~nm}$. The number of feet around the spherical center is not fixed, ranging from $4-8$, and the length of the feet is in the range 3-6 nm and their diameter is about $3 \mathrm{~nm}$.

The Pt nanostructures were analyzed by highresolution TEM (HRTEM) and electron diffraction (ED). HRTEM images of the Pt nanostructures show well defined two-dimensional lattice planes (insets of Fig. 1). The interplanar spacing is about $0.23 \mathrm{~nm}$, which can be assigned to the (111) plane of the fcc system of Pt. It has been reported that the formation of anisotropic metallic nanostructures can be attributed to the competitive growth between (111) and (100) planes [36, 57, 58]. When growth was inhibited on (111) planes, cubic particles showing the (100) planes resulted. However, when growth on (100) planes was inhibited, the resultant particles were terminated by the (111) faces. When growth on both (111) and (100) planes was inhibited, (110) planes

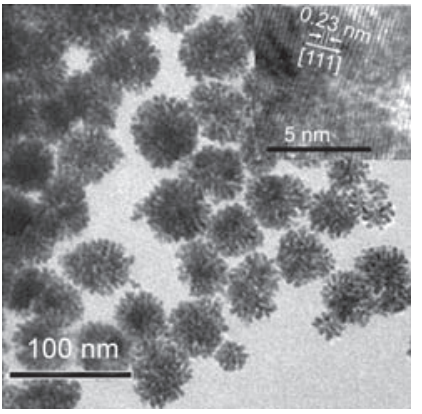

(a)

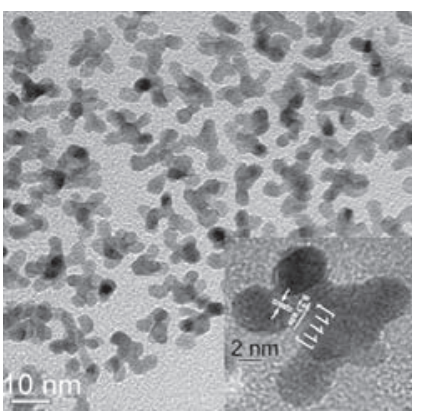

(c)

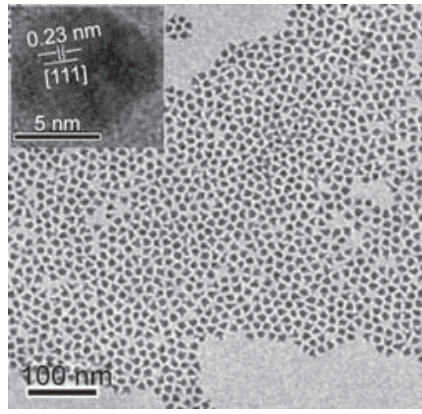

(b)

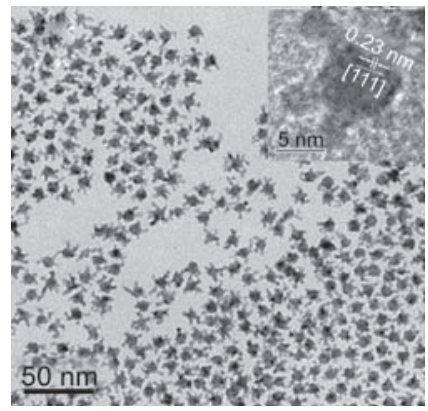

(d)

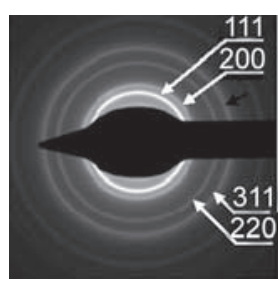

(e)
Figure 1 TEM images of Pt nanostructures synthesized in oleylamine with catalysis by $\mathrm{Au}$ nanocrystals with different amounts of Au precursor $\mathrm{AuCl}_{3}$ : (a) $3 \times 10^{-4} \mathrm{mmol}$; (b) $7.5 \times$ $10^{-4} \mathrm{mmol}$; (c) $3 \times 10^{-3} \mathrm{mmol}$; (d) $7.5 \times 10^{-3} \mathrm{mmol}$; (e) SAED pattern for a collection of Pt 
were dominant, which led to the formation of tripods. The typical selected area ED pattern for a collection of Pt nanostructures, given in Fig. 1(e), indicates that the anisotropic platinum nanostructures have a facecentered cubic (fcc) structure, in agreement with the $\mathrm{XRD}$ results.

Figure 2 shows typical XRD patterns of the $\mathrm{Pt}$ nanostructures (Pt nanostructures with different morphologies show almost identical XRD patterns). The XRD patterns reveal that the Pt nanocrystals all possess a cubic structure with high crystallinity. All of the diffraction peaks match well with Bragg reflections of the standard phase pure fcc structure of $\mathrm{Pt}$ (space group: $F m 3 m$ ), with the measured lattice constant being $a=3.92 \AA$. As expected, the width of the diffraction peaks is considerably broadened and decreases with increasing particle size. No discernible diffraction signal from gold could be observed, consistent with the very small amount of Au used. The chemical composition of the Pt nanostructures was determined using energy-dispersive X-ray (EDX) analysis. In the EDX spectrum (Fig. 3), apart from the copper and carbon signals from the TEM grid, only peaks of Pt are observed. Because no gold signal could be detected in the sample, we can say that either the $\mathrm{Au}$ concentration is too low to be detected or no $\mathrm{Au}$ is present in the Pt nanostructures.

Although there are numerous examples of

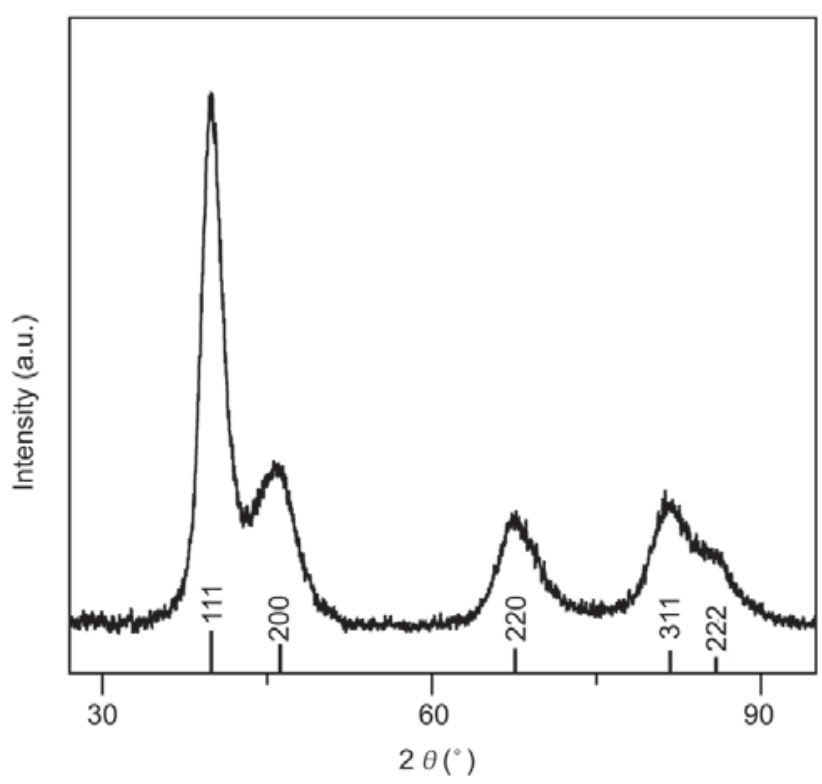

Figure 2 Typical XRD patterns of Pt nanostructures. The line XRD spectrum (bottom) corresponds to bulk cubic Pt

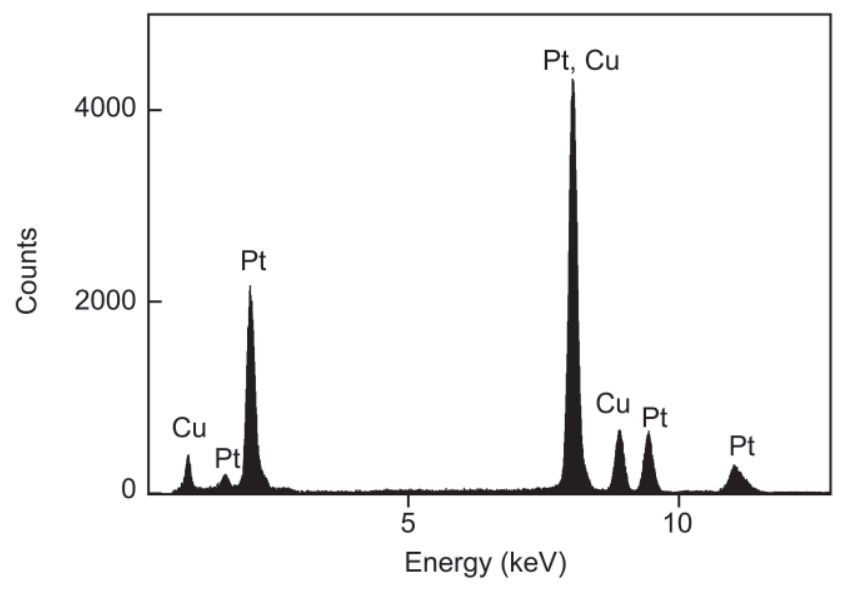

Figure 3 Representative EDX spectrum of a Pt nanostructure. The additional copper signals are contributions from the carbon-coated TEM copper grid

anisotropic shaped colloidal nanocrystals, their morphology is not easy to predict and generally varries from synthesis to synthesis. Generally speaking, the crystalline phase of the seed at the nucleating stage is critical in directing the nanocrystal shapes. Once nanocrystal seeds are formed with a specific crystalline phase, several other factors (including the intrinsic surface energies of different crystallographic surfaces, and the balance between thermodynamic and kinetic growth regimes) will affect the final geometry of the nanocrystals during the crystal growth process [7-9]. The crystal surface energy has been identified as the main driving force in the growth of anisotropic nanostructures, and the surface energy of different facets can be modulated by the use of surface-selective capping molecules. In the presence of surfactant ligands, differences in the adsorption of surfactants on the various crystal planes or differences in the steric effects of the surfactant leads to competitive growth and results in the formation of anisotropic nanostructures. In our experiments, the solvent, oleylamine plays an important role in the formation of platinum nanostructures. It acts as a reactant as well as a control agent for particle prowth and a capping reagent for architectural control. Furthermore, oleylamine can also act as a ligand and form stable complexes with $\mathrm{Pt}^{2+}$. When the temperature is raised and exceeds a critical temperature, the $\mathrm{Pt}(\mathrm{II})$ amine complex is reduced by oleylamine to zerovalent $\mathrm{Pt}^{0}$.

The trace amount of gold nanoparticles should 
play a critical role in directing the anisotropic growth of platinum nanostructures. Although no gold could be detected in the Pt nanostructure samples, we noted that no anisotropic shape formation was observed in the absence of gold seed nanoparticles. Addition of $\mathrm{AuCl}_{3}$ to the reaction mixture led to the formation of gold nanoparticles that promoted decomposition of the Pt precursors and growth of Pt nanoparticles on their low index planes. Most previous studies of metal-seeded solution phase growth of crystalline semiconductor nanowires and nanorods have been interpreted in terms of the solution-liquid-solid (SLS) mechanism proposed by Buhro et al. [40-46]. In our experiments, however, the metallic seed particles are probably not molten under the growth conditions. Thus, it is most likely that the Pt growth is catalyzed not by a liquid metal droplet but by a metal nanocrystal. Thus, we expect that the essential contribution of the seed particle is simply to provide a low-energy interface for heterogeneous nucleation of the Pt nanocrystals. The temperature required for the formation of $\mathrm{Pt}$ nanoparticles from $\mathrm{PtCl}_{2}$ via a homogeneous nucleation and growth process (i.e., without the presence of gold seed NPs) was about $240{ }^{\circ} \mathrm{C}$, while the decomposition temperature was lowered to about $180{ }^{\circ} \mathrm{C}$ when more than $25 \mu \mathrm{L} \mathrm{AuCl}_{3}$ stock solution was added, suggesting that a heterogeneous nucleation and growth process was taking place. The growth kinetics differ between the various low-index planes. The formation of Pt nanostructures with various shapes can be attributed to changes in the Pt nanocrystal nucleation and growth kinetics in the presence of the gold nanoparticles. It has been established that the monomer concentration can effectively determine the crystal morphology. According to Peng and coworkers, in the case of CdSe nanostructures in an organic phase at high temperatures, high monomer concentrations and supersaturation, and the resultant fast crystal growth are a prerequisite for formation of nonspherical nanocrystals, and spherical CdSe nanoparticles were formed at low crystal growth rates [59-65]. In contrast, Xia et al. found the opposite trend in the polyol synthesis of Pt nanostructures, where anisotropic Pt nanostructures were only formed at extremely low supersaturation in slow reduction processes $[35-39,66]$. If the reduction proceeded too quickly so that the supersaturation was too high, only isotropic, and spherical Pt nanoparticles were produced. Under our synthetic conditions, the gold seed particles can provide a low-energy interface for heterogeneous nucleation of the Pt nanocrystals and lower the activation energy for reduction of the platinum precursor and thus accelerate the growth kinetics. Furthermore, according to the fast autocatalytic mechanism [67], when Pt nuclei reach a certain size (500 atoms), the Pt nanoparticles apparently become an autocatalyst for the platinum reduction reaction. With an initiation of the reduction process, the whole process can proceed even more quickly (our results reveal that the whole process lasts less than $1 \mathrm{~min}$ ). Such fast crystal growth promotes the anisotropic growth of platinum nanostructures. The morphology control process therefore appears to be similar to that for CdSe nanocrystals, where fast crystal growth also promotes anisotropic growth [59-65].

In our system, the nanoparticles undergo higher growth rates at areas of high curvature, specifically, the corners and edges. Slower growth rates were observed at the centers of facets. This is in accordance with the diffusion control growth mechanism [68], where the diffusion flux from the bulk solution toward each growing nanocrystal surface is slower than the rate at which a growth unit is adsorbed onto the particle surface. In this situation, a gradient occurs as the supersaturation at the particle surface is low and increases with distance from the particle. If a ridge forms on the particle surface, there will be a higher supersaturation at the top of the ridge than at the base, and the incoming monomers diffusing into the diffusion sphere are mainly consumed by the ridge part, which results in an anisotropic morphology. With a spherical particle under these conditions, any perturbation of the particle surface will cause the particle edges and corners to preferentially grow. The dependence of the Pt morphologies on the concentration of $\mathrm{Au}$ seed particles results from the fact that when the concentration of seed particles is markedly increased, the same number of nucleation sites per particle leads to substantial precursor depletion. This, in turn, 
accelerates the supersaturation gradient, allowing more anisotropic growth on the particle edges and corners and resulting in the formation of irregular polyhedra, multi-branched or caterpillar-shaped particles such as those shown in Fig. 1.

\section{Conclusions}

We report an effective synthetic route to prepare anisotropic platinum nanostructures (faceted polyhedra, multi-branched rods and caterpillarshapes) via the reduction of platinum dichloride in oleylamine at intermediate temperatures with catalysis by gold seed nanoparticles. When the quantity of gold seed particles exceeds an certain value, the decomposition temperature of the platinum precursor is significantly lowered and the concentration of gold seed nanoparticles determines the morphology of the resulting platinum nanocrystals. The resulting fast crystal growth promotes the anisotropic growth of platinum nanocrystals and the results indicate that crystallite growth involves a diffusion-controlled growth process.

\section{Acknowledgment}

We thank the National Natural Science Foundation of China (Nos. 20501005, 20771037), the Program for New Century Excellent Talents in Universities of China (NCET-06-0417), Pujiang Talents Project (07pj14032), Shuguang Project (06SG33), and SRFDP (20070251014) for financial support.

Electronic Supplementary Material: Supplementary material is available in the online version of this article at http: //dx.doi.org/10.1007/s12274-008-8029-0 and is accessible free of charge.

\section{References}

[1] Schmid, G. Nanoparticles: From Theory to Application; Wiley-VCH: Weinheim, Germany, 2004.

[2] Alivisatos, A. P. Semiconductor clusters, nanocrystals, and quantum dots. Science 1996, 271, 933-937.

[3] Murray, C. B.; Kagan, C. R.; Bawendl, M. G. Synthesis and characterization of monodisperse nanocrystals and close-packed nanocrystal assemblies. Annu. Rev. Mater. Sci. 2000, 30, 545-610.

[4] Tessler, N.; Medvedev, V.; Kazes, M.; Kan, S. H.; Banin, U. Efficient near-infrared polymer nanocrystat light-emitting diodes. Science 2002, 295, 1506-1508.

[5] Sun, S.; Murray, C. B.; Weller, D.; Folks, L.; Moser, A. Monodisperse FePt nanoparticles and ferromagnetic FePt nanocrystal superlattices. Science 2000, 287, 19891992.

[6] Burda, C.; Chen, X.; Narayanan, R.; El-Sayed, M. A. Chemistry and properties of nanocrystals of different shapes. Chem. Rev. 2005, 105, 1025-1102.

[7] Jun, Y. -W.; Lee, J. -H.; Choi, J. -S.; Cheon, J. Symmetrycontrolled colloidal nanocrystals: Nonhydrolytic chemical synthesis and shape determining parameters. J. Phy. Chem. B 2005, 109, 14795-14806.

[8] Xia, Y.; Yang, P.; Sun, Y.; Wu, Y.; Mayers, B.; Gates, B.; Yin, Y.; Kim, F.; Yan, H. One-dimensional nanostructures: Synthesis, characterization, and applications. Adv. Mater. 2003, 15, 353-389.

[9] Manna, L.; Scher, E. C.; Alivisatos, A. P. Shape control of colloidal semiconductor nanocrystals. J. Cluster Sci. 2002, 13, 521-532.

[10] Wieckowski, A.; Savinova, E. R.; Vayenas, C. G. Catalysis and Electrocatalysis at Nanoparticle Surfaces; Marcel Dekker: New York, 2003.

[11] Bell, A. T. The impact of nanoscience on heterogeneous catalysis. Science 2003, 299, 1688-1691.

[12] Roucoux, A.; Schulz, J.; Patin, H. Reduced transition metal colloids: A novel family of reusable catalysts? Chem. Rev. 2002, 102, 3757-3778.

[13] Pino, L.; Recupero, V.; Beninati, S.; Shukla, A. K.; Hegde, M. S.; Bera, P. Catalytic partial-oxidation of methane on a ceria-supported platinum catalyst for application in fuel cell electric vehicles. Appl. Catal. A 2002, 225, 63-75.

[14] Williams, K. R.; Burstein, G. T. Low temperature fuel cells: Interactions between catalysts and engineering design. Catal. Today 1997, 38, 401-410.

[15] Narayanan, R.; El-Sayed, M. A. Shape-dependent catalytic activity of platinum nanoparticles in colloidal solution. Nano Lett. 2004, 4, 1343-1348.

[16] Falicov, L. M.; Somorjai, G. A. Correlation between catalytic activity and bonding and coordination number of atoms and molecules on transition metal surfaces: Theory and experimental evidence. P. Natl. Acad. Sci. USA 1985, 82, 2207-2211. 
[17] Teranishi, T.; Hosoe, M.; Tanaka, T.; Miyake, M. Size control of monodispersed Pt nanoparticles and their 2D organization by electrophoretic deposition. J. Phys. Chem. B 1999, 103, 3818-3827.

[18] Teng, X.; Liang, X.; Maksimuk, S.; Yang, H. Synthesis of porous platinum nanoparticles. Small 2006, 2, 249-253.

[19] Chen, C.-W.; Akashi, M. Synthesis, characterization, and catalytic properties of colloidal platinum nanoparticles protected by poly( $N$-isopropylacrylamide). Langmuir 1997, 13, 6465-6472.

[20] Sarathy, K. V.; Raina, G.; Yadav, R. T.; Kulkarni, G. U.; Rao, C. N. R. Thiol-derivatized nanocrystalline arrays of gold, silver, and platinum. J. Phys. Chem. B 1997, 101, 9876-9880.

[21] Zhao, S.; Chen, S.; Wang, S.; Li, D.; Ma, H. Preparation, phase transfer, and self-assembled monolayers of cubic Pt nanoparticles. Langmuir 2002, 18, 3315-3318.

[22] Yang, J.; Lee, J.; Deivaraj, T.; Too, H. An improved procedure for preparing smaller and nearly monodispersed thiol-stabilized platinum nanoparticles. Langmuir 2003, 19, 10361-10365.

[23] Chen, S.; Kimura, K. Synthesis of thiolate-stabilized platinum nanoparticles in protolytic solvents as isolable colloids. J. Phys. Chem. B 2001, 105, 5397-5403.

[24] Ahmadi, T. S.; Wang, Z. L.; Green, T. C.; Henglein, A.; El-Sayed, M. A. Shape-controlled synthesis of colloidal platinum nanoparticles. Science 1996, 272, 1924-1926.

[25] Ahmadi, T. S.; Wang, Z. L.; Henglein, A.; El-Sayed, M. A. "Cubic" colloidal platinum nanoparticles. Chem. Mater. 1996, 8, 1161-1163.

[26] Attard, G. S.; Bartlett, P. N.; Coleman, N. R. B.; Elliott, J. M.; Owen, J. R.; Wang, J. H. Mesoporous platinum films from lyotropic liquid crystalline phases. Science 1997, 278, 838-840.

[27] Song, Y.; Yang, Y.; Medforth, C. J.; Pereira, E.; Singh, A. K.; Xu, H.; Jiang, Y.; Brinker, C. J.; van Swol, F.; Shelnutt, J. A. Controlled synthesis of 2-D and 3-D dendritic platinum nanostructures. J. Am. Chem. Soc. 2004, 126, 635-645.

[28] Kijima, T.; Yoshimura T.; Uota, M.; Ikeda, T.; Fujikawa, D.; Mouri, S.; Uoyama, S. Noble-metal nanotubes (Pt, $\mathrm{Pd}, \mathrm{Ag}$ ) from lyotropic mixed-surfactant liquid-crystal templates. Angew. Chem., Int. Ed. 2004, 43, 228-232.

[29] Melosh, N. A.; Boukai, A.; Diana, F.; Gerardot, B.; Badolato, A.; Petroff, P. M.; Heath, J. R. Ultrahigh-density nanowire lattices and circuits. Science 2003, 300, 112-115.
[30] Wakayama, H.; Setoyama, N.; Fukushima, Y. Size-controlled synthesis and catalytic performance of Pt nanoparticles in micro- and mesoporous silica prepared using supercritical solvents. Adv. Mater. 2003, 15, 742-745.

[31] Shirai, M.; Igeta, K.; Arai, M. Formation of platinum nanosheets between graphite layers. Chem. Commun. 2000, 623-624

[32] Liang, H. -P.; Zhang, H. -M.; Hu, J. -S.; Guo, Y. -G.; Wan, L. -J.; Bai, C. -L. Pt hollow nanospheres: Facile synthesis and enhanced electrocatalysts. Angew. Chem., Int. Ed. 2004, 43, 1540-1543.

[33] Han, Y.; Kim, J.; Stucky, G. D. Preparation of noble metal nanowires using hexagonal mesoporous silica SBA-15. Chem. Mater. 2000, 12, 2068-2069.

[34] Shin, H. J.; Ryoo, R.; Liu, Z.; Terasaki, O. Template synthesis of asymmetrically mesostructured platinum networks. J. Am. Chem. Soc. 2001, 123, 1246-1247.

[35] Chen, J.; Herricks, T.; Geissler, M.; Xia, Y. Single-crystal nanowires of platinum can be synthesized by controlling the reaction rate of a polyol process. J. Am. Chem. Soc. 2004, 126, 10854-10855.

[36] Herricks, T.; Chen, J.; Xia, Y. Polyol synthesis of platinum nanoparticles: Control of morphology with sodium nitrate. Nano Lett. 2004, 4, 2367-2371.

[37] Chen, J.; Herricks, T.; Xia, Y. Polyol synthesis of platinum nanostructures: Control of morphology through the manipulation of reduction kinetics. Angew. Chem., Int. Ed. 2005, 44, 2589-2592.

[38] Chen, J.; Xiong, Y.; Yin, Y.; Xia, Y. Pt nanoparticles surfactant-directed assembled into colloidal spheres and used as substrates in forming Pt nanorods and nanowires. Small 2006, 2, 1340-1343.

[39] Lee, E. P; Chen, J.; Yin, Y.; Campbell, C. T.; Xia, Y. Pdcatalyzed growth of Pt nanoparticles or nanowires as dense coatings on polymeric and ceramic particulate supports. Adv. Mater. 2006, 18, 3271-3274.

[40] Trentler, T. J.; Hickman, K. M.; Goel, S. C.; Viano, A. M.; Gibbons, P. C.; Buhro, W. E. Solution-liquid-solid growth of crystalline III-V semiconductors - an analogy to vaporliquid-solid growth. Science 1995, 270, 1791-1794.

[41] Holmes, J. D.; Johnston, K. P.; Doty, R. C.; Korgel, B. A. Control of thickness and orientation of solution-grown silicon nanowires. Science 2000, 287, 1471-1473.

[42] Yu, H.; Li, J. B.; Loomis, R. A.; Wang, L. W.; Buhro, W. E. Two- versus three-dimensional quantum confinement in indium phosphide wires and dots. Nat. Mater. 2003, 2, 
517-520.

[43] Yu, H.; Buhro, W. E. Solution-liquid-solid growth of soluble GaAs nanowires. Adv. Mater. 2003, 15, 416-419.

[44] Hanrath, T.; Korgel, B. A. Nucleation and growth of germanium nanowires seeded by organic monolayercoated gold nanocrystals. J. Am. Chem. Soc. 2002, 124, 1424-1429.

[45] Yu, H.; Li, J. B.; Loomis, R. A.; Gibbons, P. C.; Wang, L. W.; Buhro, W. E. Cadmium selenide quantum wires and the transition from 3D to 2D confinement. J. Am. Chem. Soc. 2003, 125, 16168-16169.

[46] Hull, K. L.; Grebinski, J. W.; Kosel, T. H.; Kuno, M. Induced branching in confined PbSe nanowires. Chem. Mater. 2005, 17, 4416-4425.

[47] Teng, X.; Yang, H. Synthesis of platinum multipods: An induced anisotropic growth. Nano Lett. 2005, 5, 885-891.

[48] Sun, Y.; Yin, Y.; Mayers. B. T.; Herricks, T.; Xia, Y. Uniform silver nanowires synthesis by reducing $\mathrm{AgNO}_{3}$ with ethylene glycol in the presence of seeds and poly(vinyl pyrrolidone). Chem. Mater. 2002, 14, 4736-4745.

[49] Wiley, B.; Sun, Y.; Mayers, B.; Xia, Y. Shape-controlled synthesis of metal nanostructures: The case of silver. Chem. Eur. J. 2005, 11, 454-463.

[50] Zhou, S.; Mcllwrath, K.; Jackson, G.; Eichhorn B. Enhanced $\mathrm{CO}$ tolerance for hydrogen activation in Au-Pt dendritic heteroaggregate nanostructures. J. Am. Chem. Soc. 2006, 128, 1780-1781.

[51] Yong, K.-T.; Sahoo, Y.; Choudhury, K. R.; Swihart, M. T.; Minter, J. R.; Prasad, P. N. Shape control of PbSe nanocrystals using noble metal seed particles. Nano Lett. 2006, 6, 709-714.

[52] Shi, W.; Zeng, H.; Sahoo, Y.; Ohulchanskyy, T. Y.; Ding, Y.; Wang, Z. L.; Swihart, M. T.; Prasad, P. N. A general approach to binary and ternary hybrid nanocrystals. Nano Lett. 2006, 6, 875-881.

[53] Yong, K.-T.; Sahoo, Y.; Swihart, M. T.; Prasad, P. N. Growth of CdSe quantum rods and multipods seeded by noblemetal nanoparticles. Adv. Mater. 2006, 18, 1978-1982.

[54] Yong, K. -T.; Sahoo, Y.; Choudhury, K. R.; Swihart, M. T.; Minter, J. R.; Prasad, P. N. Control of the morphology and size of $\mathrm{PbS}$ nanowires using gold nanoparticles. Chem. Mater. 2006, 18, 5965-5972.

[55] Zhong, X.; Feng, Y.; Lieberwirth, I.; Knoll, W. Facile synthesis of morphology-controlled platinum nanocrystals. Chem. Mater. 2006, 18, 2468-2471.

[56] Li, J. J.; Wang, Y. A.; Guo, W.; Keay, J. C.; Mishima, T. D.;
Johnson, M. B.; Peng, X. Large-scale synthesis of nearly monodisperse $\mathrm{CdSe} / \mathrm{CdS}$ core/shell nanocrystals using airstable reagents via successive ion layer adsorption and reaction. J. Am. Chem. Soc. 2003, 125, 12567-12575.

[57] Wang, Z. L. Transmission electron microscopy of shapecontrolled nanocrystals and their assemblies. J. Phys. Chem. B 2000, 104, 1153-1175.

[58] Chen, S. H.; Wang, Z. L.; Ballato, J.; Foulger, S. H.; Carroll, D. L. Monopod, bipod, tripod, and tetrapod gold nanocrystals. J. Am. Chem. Soc. 2003, 125, 1618616187.

[59] Manna, L.; Wang, L. W.; Cingolani, R.; Alivisatos, A. P. First-principles modeling of unpassivated and surfactantpassivated bulk facets of wurtzite CdSe: A model system for studying the anisotropic growth of CdSe nanocrystals. J. Phys. Chem. B 2005, 109, 6183-6192.

[60] Scher, E. C.; Manna, L.; Alivisatos, A. P. Shape control and applications of nanocrystals. Philos. T. R. Soc. A 2003, 361, 241-255.

[61] Peng, Z. A.; Peng, X. G. Mechanisms of the shape evolution of CdSe nanocrystals. J. Am. Chem. Soc. 2001, 123, 1389-1395.

[62] Peng, Z. A.; Peng, X. G. Nearly monodisperse and shapecontrolled CdSe nanocrystals via alternative routes: Nucleation and growth. J. Am. Chem. Soc. 2002, 124, 3343-3353.

[63] Manna, L.; Scher, E. C.; Alivisatos, A. P. Synthesis of soluble and processable rod-, arrow-, teardrop-, and tetrapod-shaped CdSe nanocrystals. J. Am. Chem. Soc. 2000, 122, 12700-12706.

[64] Shieh, F.; Saunders, A. E.; Korgel, B. A. General shape control of colloidal CdS, CdSe, CdTe quantum rods and quantum rod heterostructures. J. Phys. Chem. B 2005, 109, 8538-8542.

[65] Peng, X. Mechanisms for the shape-control and shapeevolution of colloidal semiconductor nanocrystals. Adv. Mater. 2003, 15, 459-463.

[66] Zhang, Y.; Zhu, J.; Song, X.; Zhong, X. Controlling the synthesis of $\mathrm{CoO}$ nanocrystals with various morphologies.

J. Phys. Chem. C 2008, 112, 5322-5327

[67] Henglein, A.; Ershov, B. G.; Malow, M. Absorptionspectrum and some chemical-reactions of colloidal platinum in aqueous-solution J. Phys. Chem. 1995, 99, 14129-14136.

[68] Chernov, A. A. Theory of the stability of face forms of crystals. Sov. Phys.-Crystallogr. 1972, 16, 734-753.

\section{国备 Springer}

